\title{
Fasting Serum Level of Fatty-Acid-Binding Protein 4 Positively Correlates With Metabolic Syndrome in Patients With Coronary Artery Disease
}

\author{
Bang-Gee Hsu, PhD*, ; Yu-Chih Chen, $\mathrm{MD}^{\dagger+}$; Ru-Ping Lee, $\mathrm{PhD}^{* *}$; \\ Chia-Chi Lee, $\mathrm{PhD}^{\ddagger}$; Chung-Jen Lee, $\mathrm{PhD}$; Ji-Hung Wang, $\mathrm{MD}^{\dagger \dagger}$
}

\begin{abstract}
Background: The serum level of fatty-acid-binding protein 4 (FABP4) increases in patients with metabolic syndrome (MetS), so the relationship between FABP4 and MetS among patients with coronary artery disease (CAD) was investigated in the present study.

Methods and Results: Fasting blood samples were obtained from 98 CAD patients. MetS and its components were defined using the diagnostic criteria of the International Diabetes Federation; 50 CAD patients (51.0\%) had MetS. The fasting level of FABP4 positively correlated with MetS $(P=0.037)$ and serum levels of FABP4 correlated with a number of MetS criteria $(P=0.035)$. Univariate linear regression analysis showed that body fat mass $(R=0.234 ; P=0.020)$ and the levels of triglycerides $(R=0.348 ; P<0.001)$, and low-density lipoprotein-cholesterol $(R=0.217 ; P=0.032)$ positively correlated with the serum level of FABP4, whereas the level of high-density lipoprotein-cholesterol $(R=-0.243 ; P=0.016)$ negatively correlated with it. Multivariate forward stepwise linear regression analysis of the significant variables showed that the level of triglycerides $\left(\beta=0.348, R^{2}=0.121, P<0.001\right)$ was the independent predictor of fasting serum level of FABP4.
\end{abstract}

Conclusions: Among CAD patients in the present study, the fasting level of FABP4 positively correlated with MetS and serum levels of FABP4 correlated with a number of MetS criteria. (Circ J 2010; 74: 327-331)

Key Words: Coronary artery disease; Fatty-acid-binding protein 4; Metabolic syndrome

$\mathbf{T}$ he fatty-acid-binding protein 4 (FABP4; also designated aP2 or adipocyte-fatty-acid-binding protein) is expressed in adipocytes and macrophages, and integrates inflammatory and metabolic responses. ${ }^{1,2}$ Studies in FABP4-deficient mice have shown that this lipid chaperone plays a significant role in several aspects of metabolic syndrome (MetS). ${ }^{1,3}$ FABP4 affects insulin sensitivity, lipid metabolism and lipolysis, and plays an important role in atherosclerosis. ${ }^{3}$ MetS is a significant risk factor for cardiovascular disease and mortality in the general population, ${ }^{4,5}$ and is also independently associated with coronary artery disease (CAD). ${ }^{6}$ CAD patients with MetS show an increased risk of cardiovascular morbidity after follow-up. ${ }^{7}$ Recent studies have shown that the serum level of FABP4 can predict the development of MetS and is independently associated with carotid intima-media thickness. ${ }^{8} 9$ Studies of the association between the serum level of FABP4 and MetS or the number of MetS criteria in CAD patients are rare, so the aim of this study was to investigate the relationship between the fasting serum level of FABP4 and MetS among CAD patients.

\section{Methods}

Patients

Between June 2008 and December 2008, we enrolled 98 CAD patients (CAD defined as $>50 \%$ stenosis in any segment on coronary angiography) in a medical center in Hualien, eastern Taiwan (73 men, 25 women, age range 32-90 years). The Protection of Human Subjects Institutional Review Board of Tzu-Chi University and Hospital approved the study. Patients were excluded if they had any acute infections, acute myocardial infarction, pulmonary edema, heart failure at the time of blood sampling or if they refused to give informed consent for the study.

\section{Anthropometric Analysis}

Body weight was measured in light clothing and without shoes to the nearest $500 \mathrm{~g}$. Height was measured to the nearest $0.5 \mathrm{~cm}$. Waist circumference was measured to the nearest $0.5 \mathrm{~cm}$ at the shortest point below the lower rib margin and the iliac crest. Body mass index (BMI) was calculated as

Received August 4, 2009; revised manuscript received September 18, 2009; accepted September 27, 2009; released online December 14, 2009 Time for primary review: 37 days

*School of Medicine, **Department of Nursing, Tzu Chi University, ${ }^{\dagger}$ Division of Nephrology, ${ }^{\dagger}$ Division of Cardiology, Buddhist Tzu Chi General Hospital and $¥$ Department of Nursing, Tzu Chi College of Technology, Hualien, Taiwan

Mailing address: Ji-Hung Wang, MD, Division of Cardiology, Buddhist Tzu Chi General Hospital, No 707, Section 3, Chung-Yang Road, Hualen, Taiwan. E-mail: abanggeelily@yahoo.com.tw

ISSN-1346-9843 doi:10.1253/circj.CJ-09-0568

All rights are reserved to the Japanese Circulation Society. For permissions, please e-mail: cj@j-circ.or.jp 


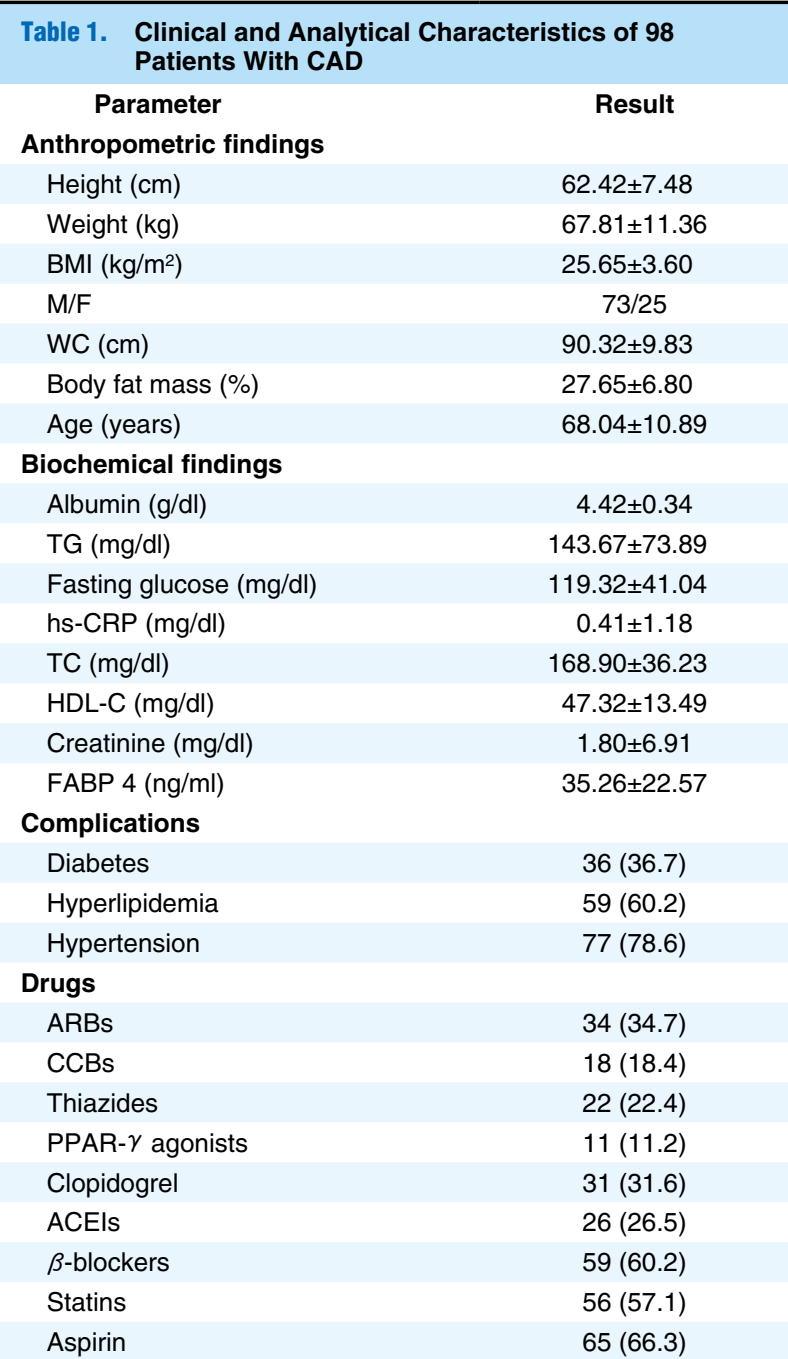

Data are mean $\pm \mathrm{SD}$ or proportion.

CAD, coronary artery disease; $\mathrm{BMI}$, body mass index; WC, waist circumference; TG, triglycerides; hs-CRP, high-sensitivity C-reactive protein; TC, total cholesterol; HDL, high-density lipoproteincholesterol; FABP 4, fatty-acid-binding protein 4; ARB, angiotensin-receptor blocker; CCB, calcium-channel blocker; PPAR, peroxisome proliferator-activated receptor; $A C E I$, angiotensinconverting enzyme inhibitor.

weight (kilograms) divided by height squared (meters). Bioimpedance measurements of fat mass were performed at the bedside according to the standard, tetrapolar, whole body (hand-foot) technique, using a single-frequency $(50-\mathrm{kHz})$ analyzer (Biodynamic-450, Biodynamics Corporation, Seattle, WA, USA). Measurements were carried out by the same operator; fat mass data were collected and analyzed by the specific formulas supplied by the manufacturer. ${ }^{10}$

\section{Biochemical Investigations}

Fasting blood samples taken from each subject were immediately centrifuged at $3,000 \times \mathrm{g}$ for $10 \mathrm{~min}$ for biochemical study. The serum was stored at $4^{\circ} \mathrm{C}$ for examination within $1 \mathrm{~h}$ of collection. Serum levels of creatinine (Cre), fasting glucose, total cholesterol, triglycerides (TG), high-density lipoproteincholesterol (HDL-C), low-density lipoprotein-cholesterol (LDL-C), albumin, globulin, and high-sensitivity C-reactive protein (hs-CRP) were measured using an autoanalyzer (COBAS Integra 800, Roche Diagnostics, Basel, Switzerland).

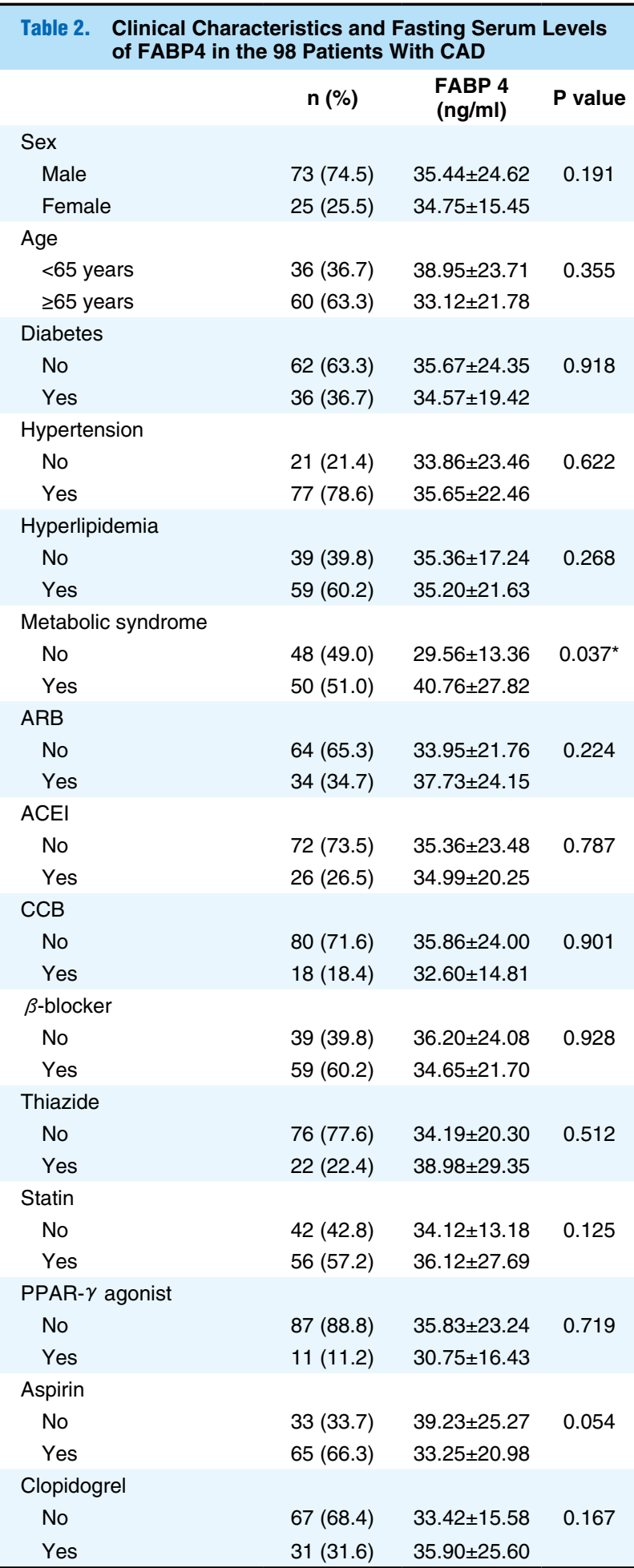

${ }^{*} \mathrm{P}<0.05$ considered statistically significant after performing the Mann-Whitney U test.

Abbreviations see in Table 1.

Serum FABP4 (SPI-BIO, Montigny le Bretonneux, France) levels were measured using a commercially available enzyme immunosassay. ${ }^{10,11}$

\section{MetS and its Components}

The prevalence of MetS was defined using the International 


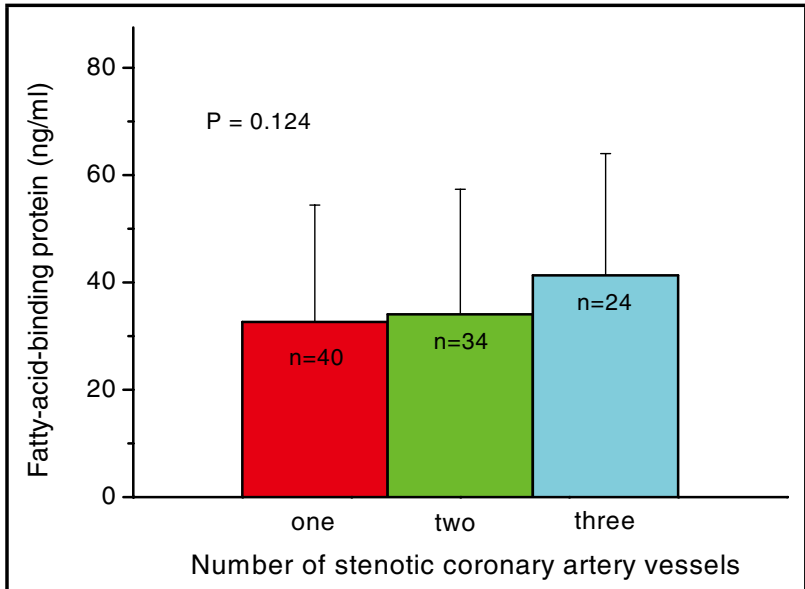

Figure 1. Fasting serum levels of FABP4 in 1, 2 and 3 stenotic coronary arteries among the 98 patients with coronary artery disease. Data were analyzed by the Kruskal-Wallis analysis of variance test.

Diabetes Federation definition. ${ }^{12}$ Subjects were classified as having MetS if they had central (abdominal) obesity with a waist circumference $\geq 90 \mathrm{~cm}$ (men) or $\geq 80 \mathrm{~cm}$ (women) (Chinese criteria), and 2 or more of the following criteria: fasting serum glucose $\geq 110 \mathrm{mg} / \mathrm{dl}$, TG $\geq 150 \mathrm{mg} / \mathrm{dl}$, HDL-C $<40 \mathrm{mg} / \mathrm{dl}$ in men or $<50 \mathrm{mg} / \mathrm{dl}$ in women, or blood pressure $\geq 130 / 85 \mathrm{mmHg}$. The use of antihypertensive medication was considered as high blood pressure in this analysis. Type 2 diabetes was determined according to World Health Organization criteria. ${ }^{13}$ A person was regarded as diabetic if the fasting plasma glucose was $\geq 126 \mathrm{mg} / \mathrm{dl}$ or the 2 -h glucose during an oral glucose tolerance test was $\geq 200 \mathrm{mg} / \mathrm{dl}$ or diabetes medication (oral or insulin) was being taken.

\section{Statistical Analysis}

Data are expressed as mean \pm standard deviation and tested for normal distribution by Kolmogorov-Smirnov statistics. Categorical variables were analyzed by the chi-square test. Comparisons between patients were performed using Student's independent t-test (2-tailed) for normally distributed data or the Mann-Whitney $U$ test for parameters that presented with non-normal distribution (fasting glucose, hsCRP, Cre, and FABP4). Clinical variables that correlated with serum FABP4 levels in the CAD patients were evaluated by univariate linear regression analyses. The significance of differences in the FABP4 level between groups (number of vessels occluded because of CAD and number of MetS criteria) was analyzed by the Kruskal-Wallis analysis of variance test. Variables that were significantly associated with FABP4 in CAD patients were tested for independency in a multivariate forward stepwise regression analysis. Data were analyzed using SPSS for Windows (version 13.0; SPSS Inc, Chicago, IL, USA). A P-value $<0.05$ was considered statistically significant.

\section{Results}

The clinical and laboratory characteristics of the CAD patients are presented in Table 1. Medical histories included diabetes $(n=36 ; 36.7 \%)$, hypertension $(n=77 ; 78.6 \%)$, and hyperlipidemia $(n=59 ; 60.2 \%)$. Drugs being used included angiotensinreceptor blockers (ARBs; $\mathrm{n}=34 ; 34.7 \%$ ), angiotensin-convert-

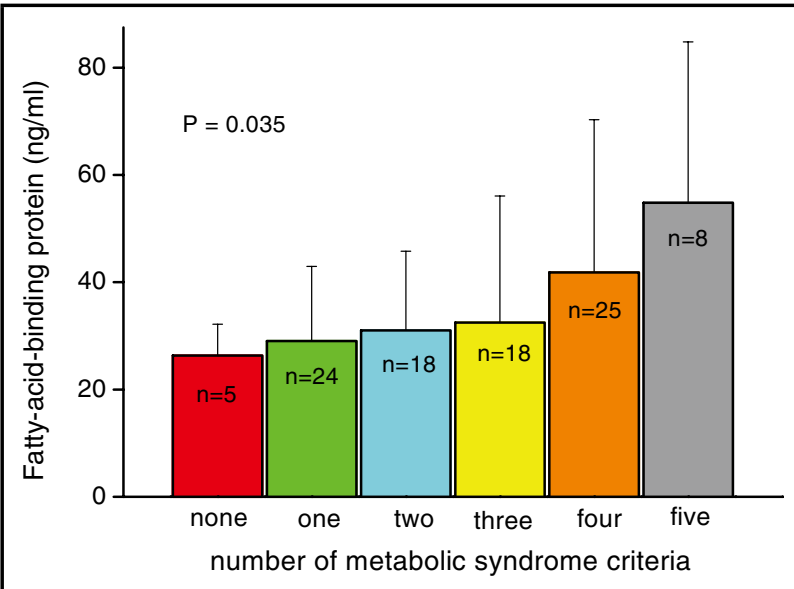

Figure 2. Fasting serum levels of FABP4 according to the number of diagnostic criteria for metabolic syndrome among the 98 patients with coronary artery disease. Data were analyzed by the Kruskal-Wallis analysis of variance test.

\begin{tabular}{|lcc|}
\hline Table 3. & \multicolumn{3}{c|}{$\begin{array}{c}\text { Correlation of Fasting Levels of Serum FABP4 and } \\
\text { Clinical Variables by Univariate Linear Analyses of } \\
\text { 98 Patients With CAD }\end{array}$} \\
S & $\beta$ & P value \\
Age (years) & -0.145 & 0.153 \\
Height $(\mathrm{cm})$ & 0.017 & 0.865 \\
Weight $(\mathrm{kg})$ & 0.154 & 0.131 \\
WC $(\mathrm{cm})$ & 0.118 & 0.249 \\
BMl $\left(\mathrm{kg} / \mathrm{m}^{2}\right)$ & 0.168 & 0.098 \\
Body fat mass (\%) & 0.234 & $0.020^{*}$ \\
Albumin (g/dl) & 0.073 & 0.476 \\
Globulin (g/dl) & -0.114 & 0.265 \\
TC (mg/dl) & 0.189 & 0.062 \\
TG (mg/dl) & 0.348 & $<0.001^{*}$ \\
HDL-C (mg/dl) & -0.243 & $0.016^{*}$ \\
LDL-C (mg/dl) & 0.217 & $0.032^{*}$ \\
Fasting glucose (mg/dl) & 0.135 & 0.186 \\
Creatinine (mg/dl) & -0.040 & 0.696 \\
Uric acid (mg/dl) & -0.098 & 0.335 \\
hs-CRP (mg/dl) & -0.062 & 0.543 \\
\hline
\end{tabular}

${ }^{*} P<0.05$ considered statistically significant after univariate linear analyses.

LDL-C, low-density lipoprotein-cholesterol. Other abbreviations see in Table 1.

ing enzyme inhibitors (ACEIs; $\mathrm{n}=26 ; 26.5 \%$ ), calcium-channel blockers (CCBs; $n=18 ; 18.4 \%), \beta$-blockers $(n=59 ; 60.2 \%)$, thiazide $(n=22 ; 22.4 \%)$ and statins $(n=56 ; 57.1 \%)$.

The clinical characteristics and fasting serum levels of FABP4 of the $98 \mathrm{CAD}$ patients are presented in Table 2: 50 patients $(51.0 \%)$ had MetS and $48(49.0 \%)$ did not. CAD patients who had MetS had higher serum fasting levels of FABP4 than those without MetS $(\mathrm{P}=0.037)$. FABP4 levels did not differ statistically by sex, age, diabetes, hypertension, hyperlipidemia, ARBs, ACEIs, CCBs, $\beta$-blockers, thiazide or statins.

Fasting serum levels of FABP4 according to the number of stenotic coronary arteries and MetS diagnostic criteria are presented in Figures 1 and 2. There was a tendency for increased fasting levels of FABP4 as the number of stenotic 
coronary arteries or diagnostic criteria for MetS increased. The highest fasting level of FABP4 occurred with 3-vessel CAD or a combination of $5 \mathrm{MetS}$ diagnostic criteria and the lowest value occurred with 1-vessel CAD vessel or no MetS diagnostic criteria. There was no statistically significant difference between the number of stenotic coronary arteries and the serum level of FABP4 $(\mathrm{P}=0.124)$. However, a statistically significant difference with the number of MetS criteria $(\mathrm{P}=0.035)$ was established.

Univariate linear analysis of the fasting serum levels of FABP4 in the CAD patients is presented in Table 3. Body fat mass $(\mathrm{R}=0.234 ; \mathrm{P}=0.020)$ and the levels of $\mathrm{TG}(\mathrm{R}=0.348$; $\mathrm{P}<0.001)$ and LDL-C $(\mathrm{R}=0.217 ; \mathrm{P}=0.032)$ positively correlated with fasting serum levels of FABP4, whereas the level of HDL-C $(\mathrm{R}=-0.243 ; \mathrm{P}=0.016)$ showed a negative correlation.

Multivariate forward stepwise linear regression analysis of the variables that were significantly associated with the fasting serum levels of FABP4 among the CAD patients (body fat mass, TG, HDL-C, LDL-C) showed that the level of TG $\left(\beta=0.348, R^{2}=0.121, P<0.001\right)$ was the independent predictor.

\section{Discussion}

The results of our study show that the fasting level of FABP4 positively associated with MetS in CAD patients, among whom the serum levels of FABP4 correlated with a number of MetS criteria and TG was an independent predictor.

MetS is a constellation of physical and laboratory abnormalities, including hypertension, hyperglycemia, hyperlipidemia, and abdominal obesity. ${ }^{12}$ Visceral fat accumulation may contribute to the development of CAD through progression of insulin resistance and an increase in apo-B-containing lipoproteins and small-sized LDL-C. ${ }^{14}$ However, another study noted that lower levels of HDL-C may be most useful for predicting CAD independent of other metabolic markers such as adiponectin, visceral fat or present medication. ${ }^{15}$ MetS constitutes a major health problem in the West and is estimated to affect at least $20 \%$ of the adult population. ${ }^{16}$ Recent studies have also demonstrated that MetS is a significant risk factor for cardiovascular disease and mortality in the general population; ${ }^{4,5}$ it is also predicted that MetS will increase the all-cause and cardiac mortality, especially after 10 years, in non-diabetic patients undergoing coronary artery bypass grafting. ${ }^{17}$ The overall prevalence of MetS in CAD patients is between $45.1 \%$ and $65.5 \% .^{7,18,19} \mathrm{In}$ our study it was $50.1 \%$, which is almost the same as reported by other studies of CAD patients. ${ }^{7}, 18$ FABP4 is a major cytoplasmic protein that is involved in the regulation of lipid metabolism and is expressed abundantly in mature adipocytes and activated macrophages. ${ }^{2,20}$ It binds fatty acid ligands with high affinity and functions in intracellular fatty acid trafficking, regulation of lipid metabolism, and modulation of gene expression. ${ }^{1,21}$ The serum level of FABP4 increases in patients with MetS, ${ }^{22,23}$ and predicts the development of MetS. ${ }^{8}$ Our study also found that CAD patients with MetS had higher fasting serum levels of FABP4. A recent study noted that FABP4 levels increase significantly as the number of stenotic coronary vessels increases from 0 to $3 .{ }^{18}$ We also noted a tendency for increasing fasting levels of FABP4 as the number of stenotic coronary vessels increased in the CAD patients; however, there was no statistically significant difference between the number of stenotic vessels and serum levels of FABP4. Moreover, our study also noted a tendency for increasing fasting levels of FABP4 as the number of diagnostic criteria for MetS increased. The highest fasting level of FABP4 occurred with a combination of 5 MetS diagnostic criteria and the lowest value was noted in patients with none.

Individuals with a fatty acid-binding protein aP2 variant have lower levels of TG and a reduced risk of coronary heart disease and obesity-induced type 2 diabetes. ${ }^{24}$ Serum levels of FABP4 positively correlate with TG and negatively correlate with HDL-C; ${ }^{21,22}$ the fasting level of TG has been identified as a significant risk factor for CAD in middle-aged Japanese men. ${ }^{25}$ In patients with diabetes, FABP4 positively correlates with plasma levels of TG, apolipoprotein C-III, and all the components of TG-rich lipoproteins, including very low-density lipoprotein (VLDL) TG, VLDL-C, and VLDL apoB ${ }^{26}$ Cabré et al also noted that plasma levels of FABP4 are increased in familial combined hyperlipidemia, ${ }^{27}$ which manifests as increased production of TG-rich lipoproteins, mainly liver-derived VLDL, with the ensuing hyperlipidemia characterized by increased plasma levels of both TG and cholesterol. ${ }^{28}$ Our study of CAD patients also noted that serum levels of TG and LDL-C positively correlated with the fasting serum level of FABP4, and that HDL-C had a negative correlation. Some studies have noted that serum levels of FABP4 positively correlate with central adiposity and fasting glucose, ${ }^{21,22}$ but we did not find such as association. FABP4 is expressed in adipocytes,,$^{1,2}$ but our study also showed that body fat mass positively correlates with the fasting serum level of FABP4. Multivariate forward stepwise linear regression analysis of the significant variables showed that in our study the TG level was the independent predictor of the fasting serum level of FABP4.

Pharmacological interventions have been shown to influence serum levels of FABP4 in humans; 3 months' treatment with atorvastatin in hyperlipidemic non-diabetic subjects substantially decreased the serum FABP4 value. ${ }^{29}$ Another study of subjects with type 2 diabetes found no difference in the levels of FABP4 in statin-treated subjects, but reported increased levels in a thiazolidinedione-treated subgroup. ${ }^{30}$ The FABP4 level was lower in subjects taking aspirin, and higher in subjects taking statins and anti-hypertensive drugs. ${ }^{18}$ Our results did not show a relationship between statins, peroxisome proliferator-activated receptor $\gamma$ agonists or other drugs (ARBs, ACEIs, CCBs or thiazide diuretics) and the serum level of FABP4. Further studies are required to elucidate the relationship between medications and the level of FABP4 in CAD patients.

\section{Study Limitations}

Firstly, this study was cross-sectional, so our findings should be investigated in long-term prospective studies before a causal relationship between the serum level of FABP4 and MetS in CAD patients can be established. Secondly, we did not have data for serum levels of insulin or the incidence of insulin resistance. Some studies have noted that the level of FABP4 positively correlates with insulin resistance or the fasting serum level of insulin. ${ }^{21,22}$ Another limitation is that we did not assess the relationship between FABP4 and MetS among patients without CAD and did not compare them with the CAD group. Further studies are needed to show the association of MetS and serum FABP4 level in patients with and without CAD.

\section{Conclusion}

We found a positive association between the circulating 
fasting level of FABP4 and MetS among CAD patients. Serum FABP4 levels correlated with the number of MetS criteria and TG was an independent predictor in this cohort.

\section{Acknowledgment}

This work was supported by grants from Tzu Chi Hospital (TCRD9832) in Taiwan.

\section{References}

1. Makowski L, Hotamisligil GS. Fatty acid binding proteins: The evolutionary crossroads of inflammatory and metabolic responses. J Nutr 2004; 134: 2464S-2468S.

2. Krusinova E, Pelikanova T. Fatty acid binding proteins in adipose tissue: A promising link between metabolic syndrome and atherosclerosis? Diabetes Res Clin Pract 2008; 82(Suppl 2): S127-S134.

3. Boord JB, Fazio S, Linton MF. Cytoplasmic fatty acid-binding proteins: Emerging roles in metabolism and atherosclerosis. Curr Opin Lipidol 2002; 13: 141-147.

4. Isomaa B, Almgren P, Tuomi T, Forsen B, Lahti K, Nissen M, et al. Cardiovascular morbidity and mortality associated with the metabolic syndrome. Diabetes Care 2001; 24: 683-689.

5. Grundy SM, Cleeman JI, Daniels SR, Donato KA, Eckel RH, Franklin BA, et al. Diagnosis and management of the metabolic syndrome: An American Heart Association/National Heart, Lung, and Blood Institute Scientific Statement. Circulation 2005; 112: $2735-2752$.

6. Anuurad E, Tracy RP, Pearson TA, Beckett L, Berglund L. Comparison of $\mathrm{C}$-reactive protein and metabolic syndrome as cardiovascular risk factors in African-Americans and EuropeanAmericans. Am J Cardiol 2009; 103: 523-527.

7. Solymoss BC, Bourassa MG, Marcil M, Levesque S, Varga S, Campeau L. Long-term rates of cardiovascular events in patients with the metabolic syndrome according to severity of coronaryangiographic alterations. Coron Artery Dis 2009; 20: $1-8$.

8. Xu A, Tso AW, Cheung BM, Wang Y, Wat NM, Fong CH, et al. Circulating adipocyte fatty acid binding protein levels predict the development of the metabolic syndrome: A 5-year prospective study. Circulation 2007; 115: 1537-1543.

9. Yeung DC, Xu A, Cheung CW, Wat NM, Yau MH, Fong CH, et al. Serum Adipocyte fatty acid-binding protein levels were independently associated with carotid atherosclerosis. Arterioscler Thromb Vasc Biol 2007; 27: 1796-1802.

10. Lee CC, Lee RP, Subeq YM, Wang CH, Fang TC, Hsu BG. Fasting serum total ghrelin level inversely correlates with metabolic syndrome in hemodialysis patients. Arch Med Res 2008; 39: 785-790.

11. Wang JH, Subeq YM, Tsai WC, Lee RP, Hsu BG. Intravenous Nacetylcysteine improves renal function and ameliorates plasma total homocysteine in patients undergoing cardiac angiography. Ren Fail 2008; 30: $527-533$.

12. Alberti KG, Zimmet PZ, Shaw J. Metabolic syndrome: A new world-wide definition: A consensus statement from the International Diabetes Federation. Diabet Med 2006; 23: 469-480.

13. Alberti KG, Zimmet PZ. Definition, diagnosis and classification of diabetes mellitus and its complications. I: Diagnosis and classification of diabetes mellitus provisional report of a WHO consultation. Diabet Med 1998; 15: 539-553.

14. Kobayashi H, Nakamura T, Miyaoka K, Nishida M, Funahashi T,
Yamashita S, et al. Visceral fat accumulation contributes to insulin resistance, small-sized low-density lipoprotein, and progression of coronary artery disease in middle-aged non-obese Japanese men. Circ J 2001; 65: 193-199.

15. Mitsutake R, Miura S, Kawamura A, Saku K. Are metabolic factors associated with coronary artery stenosis on MDCT? Circ J 2009; 73: $132-138$.

16. Scott CL. Diagnosis, prevention, and intervention for the metabolic syndrome. Am J Cardiol 2003; 92: 35i-42i.

17. Kajimoto $\mathrm{K}$, Kasai $\mathrm{T}$, Miyauchi $\mathrm{K}$, Hirose $\mathrm{H}$, Yanagisawa N, Yamamoto T, et al. Metabolic syndrome predicts 10-year mortality in non-diabetic patients following coronary artery bypass surgery. Circ J 2008; 72: 1481 - 1486 .

18. Rhee EJ, Lee WY, Park CY, Oh KW, Kim BJ, Sung KC, et al. The association of serum adipocyte fatty acid-binding protein with coronary artery disease in Korean adults. Eur J Endocrinol 2009; 160: $165-172$.

19. Anuurad E, Chiem A, Pearson TA, Berglund L. Metabolic syndrome components in African-Americans and European-American patients and its relation to coronary artery disease. Am J Cardiol 2007; 100: $830-834$.

20. Furuhashi M, Hotamisligil GS. Fatty acid-binding proteins: Role in metabolic diseases and potential as drug targets. Nat Rev Drug Discov 2008; 7: 489-503.

21. Makowski L, Hotamisligil GS. The role of fatty acid binding proteins in metabolic syndrome and atherosclerosis. Curr Opin Lipidol 2005; 16: $543-548$.

22. Stejskal D, Karpisek M. Adipocyte fatty acid binding protein in a Caucasian population: A new marker of metabolic syndrome? Eur J Clin Invest 2006; 36: 621-625.

23. Xu A, Wang Y, Xu JY, Stejskal D, Tam S, Zhang J, et al. Adipocyte fatty acid binding protein is a plasma biomarker closely associated with obesity and metabolic syndrome. Clin Chem 2006; 52: 405413.

24. Tuncman G, Erbay E, Hom X, De Vivo I, Campos H, Rimm EB, et al. A genetic variant at the fatty acid-binding protein aP2 locus reduces the risk for hypertriglyceridemia, type 2 diabetes, and cardiovascular disease. Proc Natl Acad Sci USA 2006; 103: 6970_ 6975.

25. Satoh $\mathrm{H}$, Nishino T, Tomita K, Tsutsui H. Fasting triglyceride is a significant risk factor for coronary artery disease in middle-aged Japanese men. Circ J 2006; 70: 227-231.

26. Cabré A, Lázaro I, Girona J, Manzanares JM, Marimón F, Plana N, Heras M, et al. Plasma fatty acid binding protein 4 is associated with atherogenic dyslipidemia in diabetes. J Lipid Res 2008; 49: $1746-1751$.

27. Cabré A, Lázaro I, Cofan M, Jarauta E, Plana N, Garcia-Otin AL, et al. FABP4 plasma levels are increased in familial combined hyperlipidemia. J Lipid Res 2009 (in press)

28. Wierzbicki AS, Graham CA, Young IS, Nicholls DP. Familial combined hyperlipidaemia: Under-defined and under-diagnosed? Curr Vasc Pharmacol 2008; 6: 13-22.

29. Karpisek M, Stejskal D, Kotolova H, Kollar P, Janoutova G, Ochmanova $\mathrm{R}$, et al. Treatment with atorvastatin reduces serum adipocyte-fatty acid binding protein value in patients with hyperlipidaemia. Eur J Clin Invest 2007; 37: 637-642.

30. Cabré A, Lázaro I, Girona J, Manzanares JM, Marimón F, Plana N, et al. Fatty acid binding protein 4 is increased in metabolic syndrome and with thiazolidinedione treatment in diabetic patients. Atherosclerosis 2007; 195: e150-e158. 